\title{
Radiofrequency Ablation of Subpleural Lung Malignancy: Reduced Pain Using an Artificially Created Pneumothorax
}

\author{
Edward W. Lee $\cdot$ Robert D. Suh · Michelle R. Zeidler • \\ Irene S. Tsai - Robert B. Cameron . \\ Fereidoun G. Abtin · Jonathan G. Goldin
}

Received: 5 August 2008/Accepted: 8 January 2009/Published online: 5 February 2009

(c) The Author(s) 2009. This article is published with open access at Springerlink.com

\begin{abstract}
One of the main issues with radiofrequency (RF) ablation of the subpleural lung malignancy is pain management during and after RF ablation. In this article, we present a case that utilized a technique to decrease the pain associated with RF ablation of a malignancy located within the subpleural lung. Under CT guidance, we created an artificial pneumothorax prior to the RF ablation, which resulted in minimizing the pain usually experienced during and after the procedure. It also decreased the amount of pain medications usually used in patients undergoing RF ablation of a subpleural lung lesion.
\end{abstract}

Keywords Radiofrequency ablation .

Subpleural lung malignancy $\cdot$ Iatrogenic pneumothorax

E. W. Lee $\cdot$ R. D. Suh $(\bowtie) \cdot$ I. S. Tsai .

F. G. Abtin · J. G. Goldin

Department of Radiological Sciences, Thoracic Imaging

and Intervention, Center for the Health Sciences (CHS), UCLA

Medical Center, David Geffen School of Medicine at UCLA,

10833 Le Conte Avenue, B2-168, Los Angeles 90095-1721,

CA, USA

e-mail: rsuh@mednet.ucla.edu

E. W. Lee

e-mail: EdwardLee@mednet.ucla.edu

M. R. Zeidler

Department of Internal Medicine, Pulmonary and Critical Care, David Geffen School of Medicine at UCLA, Los Angeles, CA, USA

R. B. Cameron

Department of Surgery, Thoracic Oncology, David Geffen

School of Medicine at UCLA, Los Angeles, CA, USA

\section{Introduction}

Percutaneous radiofrequency (RF) ablation is a minimally invasive and nonsurgical technique used to treat various solid tumors. Many studies have shown that this technique is safe and effective in treating hepatic and renal tumors [1-3]. Recent investigations of RF ablation's practicability and outcome as a successful method for extricating primary and metastatic lung tumors have been promising [4-8]. Studies have shown that RF ablation is evolving into a suitable alternative and adjuvant method to open thoracic surgery of primary and metastatic lung cancer, which is commonly associated with high morbidity and mortality $[9$, 10]. However, there are many issues concerning RF ablation that need to be addressed. These questions mainly involve patient selection, location and type of lung malignancy, accessibility of the lesion, possible risks for acute and chronic complications and management, and finally, pain management, both during and after the RF ablation [11]. In this article, we present a case that utilized a technique to decrease the pain associated with RF ablation of a malignancy located within the subpleural lung. Under CT guidance, we created an artificial pneumothorax prior to the RF ablation, which resulted in minimizing the pain usually experienced during and after the procedure.

\section{Case Report}

The patient's written informed consent was obtained after the risk, benefits, and alternatives of the procedure were fully explained. A waiver of informed consent was obtained from the IRB for this report.

A 43-year-old woman with a history of metastatic colorectal cancer to the liver, originally diagnosed in 1999 , 
presented with newly rising carcinoembryogenic antigen (CEA), and CT scan of the chest confirmed the development of three new subcentimeter nodules within both lungs. These nodules were consistent with pulmonary parenchymal metastases: one within the right middle lobe and, notably, two nodules within the anterior segment of the left upper lobe, one within the subpleural aspect of the lobe (Fig. 1A) and the other central. Due to slow progression of the tumor within the lung, complications of hypercoagulability related to the patient's current systemic chemotherapy and her prior familiarity with RF ablation for at least three hepatic metastases, RF ablation was preferred over surgical metastasectomy.

The patient was prepped and draped in sterile fashion, and midazolam hydrochlorate $(1 \mathrm{mg}$ of Versed; Roche Laboratories, Nutley, NJ, USA), fentanyl citrate ( $25 \mu \mathrm{g}$ of Sublimaze; Akorn, Buffalo Grove, IL, USA), and diphenhydramine hydrochloride (25 mg of Benadryl; Pfizer Inc., New London, CT, USA) were administered intravenously to induce moderate sedation. Utilizing the GE HiSpeed Advantage CT Scanner (GE Medical Systems, Milwaukee, WI, USA) with the patient in the supine position, a 22gauge Chiba needle (Cook, Bloomington, IN, USA) was placed adjacent to the peripherally located metastasis within the anterior segment of the left upper lobe via anterior intercostal approach (Fig. 1A). Following CT confirmation of the needle tip, a RITA 6.4-Fr Starburst-XL multiarray electrode (RITA Medical Systems, Mountain View, CA, USA) was placed so that its leading tip was situated to enter proximally into the parietal and visceral pleura and the underlying lung.

Based on results by others using iatrogenic pneumothorax for easy access to a tumor [12-14], we deduced that iatrogenic pneumothorax may decrease pain by separating the two pleural layers or the visceral from the somatically innervated parietal pleura. With slow and deliberate tine deployment to $3 \mathrm{~cm}$, an intentional and iatrogenic artificial pneumothorax was created, the tines of the electrode piercing the lung and surrounding the targeted nodule, while displacing the visceral pleura away from the parietal pleura (Fig. 1B). In order to further enlarge the potential pneumothorax, the deployed electrode was then advanced, ensuring that its $1-\mathrm{cm}$ active tip was entirely within the pleural space and pneumothorax (Fig. 1C). Subsequently, a single RF ablation was performed according to the manufacturer's guide (Fig. 1C). By separating the visceral and somatically innervated parietal pleura with a potential space, we observed very minimal patient discomfort during the ablation session. In addition, we were still able to achieve effective capture of the targeted nodule within the expanded array, without negatively affecting the patient's oxygen saturation and vital signs. Following completion of the ablation, the tines were retracted with the electrode tip still residing within the pleural space and pneumothorax. With a 20-ml Luer lock syringe attached to the side port of the electrode, the RF electrode was withdrawn with negative suction applied to the syringe, thereby manually decompressing the associated localized pneumothorax and actively aspirating any potential air leak (Fig. 1D). The second nodule was then localized with the Chiba needle, and via tandem needle technique, the Starburst electrode was driven to the nodule, its tines deployed, and the metastasis ablated using standard technique. Due to the second nodule's more central location, repeat creation of an artificial pneumothorax was not performed. Before RF ablation, as the Chiba needle was removed, $2 \mathrm{ml}$ of

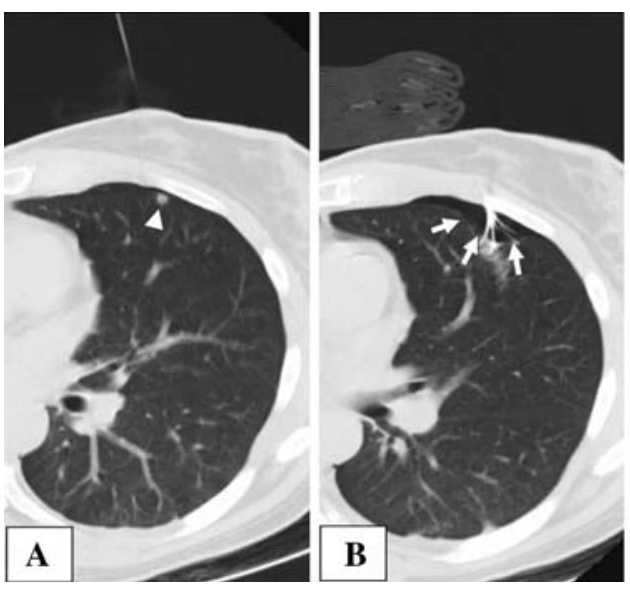

Fig. 1 Sequential CT images of radiofrequency ablation of subpleural tumor. A A 22-gauge Chiba needle was placed adjacent to the peripherally located metastasis (white arrowhead: subpleural tumor). B An intentional and iatrogenic artificial pneumothorax was created displacing the visceral pleura (white arrows: artificial pneumothorax).

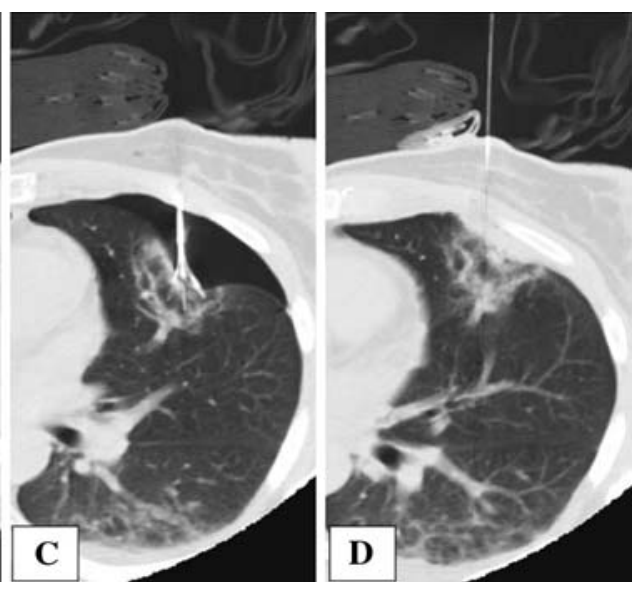

C Enlargement of the artificially created pneumothorax further separated the ablation site from the parietal pleura. D Following completion of the ablation, the tines were retracted and a localized pneumothorax was decompressed by active aspiration using a syringe 
autologous blood was injected along its parenchymal tract. Intraprocedural CT scans of the thorax showed no air within the pleural cavity following the first ablation and a minimally sized residual pneumothorax following the second ablation, which required no subsequent intervention or further treatment. The overall procedure time was not significantly different from that for the conventional method. Postablation CT images demonstrated progressive ground-glass attenuation envelopment of each targeted nodule, suggestive of a successful RF ablation. Throughout the RF ablation procedure and postprocedure observation period, the patient's vital signs, including oxygen saturation, remained stable. Following the procedure, the patient did not require any additional analgesia or narcotics for pain control. The patient was discharged on the same day with minimal oral NSAID for pain control.

Nine-month follow-up CT images demonstrated dense bands of scarring with signs of shrinkage of ablationinduced areas of necrosis, as well as complete resolution of the pneumothorax.

\section{Discussion}

$\mathrm{RF}$ ablation is a minimally invasive and effective method used to treat various tumors by inducing complete coagulation necrosis and cell death of a targeted tumor, with generally few complications. The physiological heat insulation created by air surrounding the tumor produces more effective thermal energy for complete cell necrosis [15]; therefore, RF ablation is thought to be a suitable treatment option for pulmonary malignancy. However, the literature on the percutaneous ablation of thoracic tumors, although expanding, is still incomplete, and multiple technical issues require address and optimization.

One issue that has been a constant challenge for RF operators has been patient pain management. For the most part, RF ablation has been performed as an outpatient procedure, usually under moderate sedation. Operators have at times favored deep sedation and even general anesthesia, particularly in patients with targeted lesions involving the pleura and/or chest wall, and especially in those seeking palliation for pain. RF ablation-induced pain causes discomfort and agitation for patients during and following the procedure. It can cause a wide spectrum of pain ranging from tolerably mild pain to severe pain requiring high doses of narcotics or sedatives, or even general anesthesia, which necessitates anesthesiology service. One study has shown that $R F$ ablation-induced pain is the second most frequently cited complication of the RF ablation procedure, requiring general anesthesia at their institution [16]. In particular, RF ablation involving the pleural margin, as in our case, has been known to cause more severe pain, usually requiring multiple intercostal nerve blocks or general anesthesia to proceed with the procedure $[11,17]$.

The consequences of RF ablation-induced pain can present additional problematic issues. With severe intraablational pain and its management, subsequent electrode repositioning for overlapping ablations required in larger tumor therapy may be jeopardized, in that a higher and deeper level of moderate sedation and pain control during the procedure will diminish lung aeration and expansion and patient cooperation. The technical difficulties encountered in repeated electrode repositioning and deployment may overly lengthen the procedure and induce additional complications, especially in those patients with advanced lung diseases and poor pulmonary reserve, leading to difficult and prolonged recovery.

With prolonged postprocedural pain, patients may splint and limit their respiratory excursion. With decreased lung ventilation and clearance function, the possibility of infection rises within the suboptimally aerated lung. In general, RF ablation-induced pain reduces the benefits of the noninvasiveness of RF ablation and may cause relatively extended care and potential hospitalization for post$\mathrm{RF}$ ablation pain control. Therefore, the procedure may become less cost-effective and less attractive as an alternative therapeutic method to surgery.

As previously mentioned, RF ablation of tumors involving the pleural margin is known to incite more pain sensors than others [11]. However, our case presentation has suggested that an artificially created pneumothorax may mitigate the pain for those patients undergoing $\mathrm{RF}$ ablation of those types of tumors. In our patient, the amount of sedation $(25 \mu \mathrm{g}$ of fentanyl and $1 \mathrm{mg}$ of midazolam) used was substantially less than what is usually required (200 $\mu \mathrm{g}$ of fentanyl and $3 \mathrm{mg}$ of midazolam) in our experience. In fact, this technique does not require general anesthesia or intercostal nerve block. Despite creation of a pneumothorax, the overall procedure time was not significantly increased, since the pneumothorax was established as the electrode was inserted and not beforehand. Overall, complications related to intraprocedural pain and deep sedation are minimized, potentially with better outcomes than with the other conventional methods of treating subpleural tumors. Although our case may suggest benefits of using iatrogenic pneumothorax to reduce the pain, possible risks and limitation must be evaluated. No major unpredicted complications are expected other than the usual potential complications that RF ablation of the lung may cause. However, a larger than planned pneumothorax or a pneumothorax related to an actual air leak may be anticipated. This may cause respiratory distress, but immediate awareness and treatment should minimize further deleterious respiratory 
deterioration. There are reports of utilizing artificially created pneumothorax to reach tumors in other target organs, such as the upper pole of the kidney [12], the hepatic dome [13], and the mediastinum/pulmonary hilum [14] to prevent traversal of aerated lung parenchyma. However, this is one of the first observations of utilizing an artificial pneumothorax to reduce RF ablation-induced pain in ablation of an intrathoracic intrapulmonary subpleural tumor. Future investigation focused on followings will be useful to potentiate the pain-reducing effect of iatrogenic pneumothorax in lung RF ablation: (i) evaluating a cohort of patients utilizing a subjective and objective pain scale, both during and after the procedure; and (ii) investigating how reproducible and feasible this technique is in different clinical settings such as patients with a history of lung/ pleural surgery with scarring or patients with emphysematous disease.

Open Access This article is distributed under the terms of the Creative Commons Attribution Noncommercial License which permits any noncommercial use, distribution, and reproduction in any medium, provided the original author(s) and source are credited.

\section{References}

1. Dupuy DE, Goldberg SN (2001) Image-guided radiofrequency tumor ablation: challenges and opportunities-part II. J Vasc Interv Radiol 12:1135-1148

2. Nahum Goldberg S, Dupuy DE (2001) Image-guided radiofrequency tumor ablation: challenges and opportunities-part I. J Vasc Interv Radiol 12:1021-1032

3. Brown DB (2005) Concepts, considerations, and concerns on the cutting edge of radiofrequency ablation. J Vasc Interv Radiol 16:597-613

4. Dupuy DE, Mayo-Smith WW, Abbott GF, DiPetrillo T (2002) Clinical applications of radio-frequency tumor ablation in the thorax. Radiographics 22(Special issue):S259-S269

5. Nishida T, Inoue K, Kawata Y et al (2002) Percutaneous radiofrequency ablation of lung neoplasms: a minimally invasive strategy for inoperable patients. J Am Coll Surg 195:426-430
6. Steinke K, King J, Glenn D, Morris DL (2003) Radiologic appearance and complications of percutaneous computed tomography-guided radiofrequency-ablated pulmonary metastases from colorectal carcinoma. J Comput Assist Tomogr 27: 750-757

7. Jin GY, Lee JM, Lee YC, Han YM, Lim YS (2004) Primary and secondary lung malignancies treated with percutaneous radiofrequency ablation: evaluation with follow-up helical CT. AJR 183:1013-1020

8. Suh RD, Wallace AB, Sheehan RE, Heinze SB, Goldin JG (2003) Unresectable pulmonary malignancies: CT-guided percutaneous radiofrequency ablation-preliminary results. Radiology 229: 821-829

9. Ferguson MK, Durkin AE (2003) A comparison of three scoring systems for predicting complications after major lung resection. Eur J Cardiothorac Surg 23:35-42

10. Mazzone PJ, Arroliga AC (2005) Lung cancer: preoperative pulmonary evaluation of the lung resection candidate. Am J Med 118:578-583

11. VanSonnenberg E, Shankar S, Morrison PR et al (2005) Radiofrequency ablation of thoracic lesions: part 2. Initial clinical experience-technical and multidisciplinary considerations in 30 patients. AJR Am J Roentgenol 184:381-390

12. Ahrar K, Matin S, Wallace MJ, Gupta S, Hicks ME (2005) Percutaneous transthoracic radiofrequency ablation of renal tumors using an iatrogenic pneumothorax. AJR Am J Roentgenol 185:86-88

13. de Baere T, Dromain C, Lapeyre M et al (2005) Artificially induced pneumothorax for percutaneous transthoracic radiofrequency ablation of tumors in the hepatic dome: initial experience. Radiology 236:666-670

14. Scalzetti EM (2005) Protective pneumothorax for needle biopsy of mediastinum and pulmonary hilum. J Thorac Imaging 20: 214-219

15. Lencioni R, Crocetti L, Cioni R et al (2004) Radiofrequency ablation of lung malignancies: where do we stand? Cardiovasc Interv Radiol 27:581-590

16. Gadaleta C, Mattioli V, Colucci G et al (2004) Radiofrequency ablation of 40 lung neoplasms: preliminary results. AJR Am J Roentgenol 183:361-368

17. Steinke K, King J, Glenn DW, Morris DL (2004) Percutaneous radiofrequency ablation of lung tumors with expandable needle electrodes: tips from preliminary experience. AJR Am J Roentgenol 183:605-611 